\title{
Percepciones de docentes de Educación Primaria participantes en el programa Andalucía Profundiza sobre el aprendizaje cooperativo y por investigación
}

\author{
Cristina García-Ruiz, Teresa Lupión-Cobos y Ángel Blanco-López \\ Universidad de Málaga, Facultad de Ciencias de la Educación, \\ Didáctica de las ciencias Experimentales, Málaga. España
}

[Recibido el 16 de mayo de 2020, aceptado el 13 de julio de 2020]

En este trabajo se analizan las percepciones de maestros/as de Educación Primaria en ejercicio participantes en un programa institucional de innovación educativa denominado Andalucía Profundiza, sobre el aprendizaje cooperativo y por investigación como una metodología activa en la enseñanza de las ciencias. Para la recogida de datos se ha utilizado un cuestionario adaptado del proyecto europeo PRIMAS. Los resultados obtenidos muestran que los beneficios asociados a este tipo de metodologías pueden suponer una ventaja a la hora de ser incorporadas en los proyectos del programa Andalucía Profundiza, si bien todavía requieren de un proceso de concienciación para ser implementadas por el profesorado en ejercicio.

Palabras clave: Percepciones de maestros/as en ejercicio; Educación Primaria; Aprendizaje cooperativo; Proyectos de investigación; Programa Andalucía Profundiza.

\section{Perceptions of Primary Education teachers participating in the Andalucía Profundiza programme about cooperative and inquiry learning}

This paper analyzes the perceptions of in-service Primary Education teachers participating in an institutional programme of educational innovation called Andalucía Profundiza, on the use of inquiry cooperative learning as an active methodology in science teaching. A questionnaire adapted from the PRIMAS European project has been used for data collection. The results show that the benefits associated with this type of methodologies can be an advantage when they are incorporated into the Andalucía Profundiza projects. However, they still require an awareness process to be implemented by in-service teachers.

Keywords: In-service elementary teachers' beliefs; Primary Education; Cooperative learning; Inquiry Projects; Andalucía Profundiza programme.

Para citar el artículo. García-Ruiz, C., Lupión-Cobos, T. y Blanco-López, A. (2020). Percepciones de docentes de Educación Primaria participantes en el programa Andalucía Profundiza sobre el aprendizaje cooperativo y por investigación. Ápice. Revista de Educación Científica, 4 (2), 1-15. DOI: https://doi.org/10.17979/arec.2020.4.2.6536

Contacto.crisgarcia@uma.es, teluco@uma.es, ablancol@uma.es 


\section{Introducción}

Las necesidades de aprendizaje del siglo XXI requieren del uso de nuevas líneas metodológicas que favorezcan el proceso de enseñanza-aprendizaje hacia la generación de un conocimiento compartido, en el que el alumnado se convierta en el centro de su propio aprendizaje. Esto supone que el profesorado en ejercicio ha de abordar nuevos retos docentes mediante la búsqueda de dinámicas y propuestas innovadoras, tarea a la que contribuyen los programas de innovación institucionales. En concreto, las técnicas de trabajo cooperativo, que a menudo aparecen asociadas a este tipo de programas, se establecen como dinámicas favorecedoras en el aula (Fernández-Lozano et al., 2012), permitiendo la implicación del alumnado como "investigador novel", enriqueciendo su actividad mediante un tratamiento científico-tecnológico de los problemas y abordando la puesta en práctica de estrategias de resolución a través de diferentes perspectivas. No obstante, su aplicación puede resultar un reto docente (Lupión-Cobos y Martín-Gámez, 2016), ya que requiere un diseño cuidadoso de las actividades a desarrollar y su posterior enriquecimiento, para asegurar así la implicación del alumnado en los problemas planteados.

Considerando de gran interés conocer el impacto real de la aplicación de este tipo de enfoques en la calidad docente, este trabajo pretende contribuir a la identificación de las principales dificultades encontradas por el profesorado en activo, en su práctica de estrategias cooperativas y de investigación dentro del marco de los proyectos del programa institucional Andalucía Profundiza. Escenario en el que abordamos las siguientes preguntas de investigación:

1. ¿Qué percepciones muestran los maestros y maestras en ejercicio participantes en el programa Andalucía Profundiza sobre el aprendizaje cooperativo y por investigación como metodología activa de enseñanza-aprendizaje?

2. ¿Existe alguna relación entre estas percepciones y el perfil profesional docente específico de los participantes en el programa?

\section{Marco teórico}

\section{Enseñanza de la ciencia mediante el enfoque de investigación}

El acercamiento de la ciencia a las aulas requiere de la adecuada combinación de diferentes prácticas epistémicas y sociales compartidas con la comunidad científica (Osborne, 2011). Una de las formas de aunarlos es a través de proyectos de investigación, que resultan apropiados no solo para incrementar el grado de motivación hacia el aprendizaje, sino, también, para mejorar el aprendizaje de conocimientos y el desarrollo de habilidades (Song, 2018), aspectos que se recogen ampliamente en la literatura (Comeaux y Huber, 2001; Domènech-Casal, 2018; Mioduser y Betzer, 2008; Osborne y Dillon, 2008).

El interés por la enseñanza-aprendizaje de la ciencia a través del enfoque de investigación aumentó considerablemente a principios de la década de 1990, momento a partir del cuál los conceptos de indagación, realización de experimentos o actividades prácticas y participación activa, comenzaron a formar parte de metodologías activas como instrumento para estimular la alfabetización científica (Sjøberg, 2019), promocionadas en la mayoría de políticas e iniciativas internacionales sobre educación científica (Durando et al., 2019).

Las ventajas asociadas a este enfoque de enseñanza de las ciencias por investigación (también llamado de indagación), que se encuentra en línea con los principios propios de las teorías de aprendizaje de tipo constructivistas, contemplan, entre otros aspectos, una mejora de la motivación y el interés hacia el aprendizaje, disminución de la brecha de 
género, o aumento de las vocaciones científicas, creando además un efecto duradero en las actitudes hacia la ciencia (Durando et al., 2019).

Sin embargo, a pesar de ser una de las estrategias más habituales para involucrar al alumnado en los procesos de integración y construcción del conocimiento científico (Rocard et al., 2007), tradicionalmente, se ha llevado a cabo en forma de "receta", con los docentes proporcionando instrucciones precisas y estructuradas a su alumnado para seguir el protocolo, obviando así la formulación de preguntas, la planificación del diseño experimental o la discusión de los resultados, aspectos clave en las prácticas científicas. Esta identificación errónea del procedimiento de investigación evidencia un distanciamiento de lo que se entiende, de manera muy generalizada, como actividad de investigación o indagación versus actividades prácticas. Asimismo, a menudo no se interpreta que la actividad indagatoria pueda no conllevar, necesariamente, actividades prácticas (Couso, 2014; Pérez y Furman, 2016).

\section{La investigación y el uso de estrategias cooperativas}

En el área de la Didáctica de las Ciencias Experimentales, la investigación educativa entorno a los principales problemas asociados al proceso de enseñanza-aprendizaje de las ciencias apoya el uso de estrategias de trabajo cooperativo para implicar a los estudiantes en la construcción de su propio aprendizaje, a través de un aprendizaje por indagación cooperativo que acerque a los estudiantes al rol investigador (Woods-McConney et al., 2016).

En las últimas décadas han proliferado publicaciones que recogen las bondades del trabajo cooperativo, cuya base teórica radica en las teorías del constructivismo social que enfatizan el aprendizaje a través de las interacciones sociales (Vigotsky, 1978), y que, sin duda han contribuido a la renovación pedagógica de la educación. Desde estos planteamientos, en los proyectos de investigación, el alumnado, trabajando en pequeños grupos de forma conjunta, comparte y discute información a través de interacciones mutuas (Ucan y Webb, 2015). Se produce así una primera aproximación al trabajo científico, mediada por un papel activo del alumnado, desde el que aprenden a hablar y razonar juntos de manera efectiva, razonando sobre diferentes problemas, y planteando y respondiendo preguntas (Gillies et al., 2013).

Este acercamiento al mundo científico significa involucrar al alumnado en la resolución del problema abordándolo desde múltiples perspectivas y estrategias, incluyendo como procesos la exploración y comprensión del problema, la planificación, ejecución y monitorización de la investigación o la reflexión de los resultados (Organisation for Economic Co-operation and Development [OECD], 2017).

En la mayoría de los casos, este proceso implica trabajar de forma cooperativa hacia un objetivo común (Hesse et al., 2015), utilizando para ello habilidades sociales y cognitivas que ayuden a desarrollar una comprensión compartida, tomar las medidas adecuadas y establecer y mantener una organización de equipo para resolver el problema (Fiore et al., 2010). Las características intrínsecas del trabajo cooperativo (interdependencia positiva, interacción promotora, responsabilidad personal e individual, habilidades interpersonales y de grupo y procesamiento grupal o autoevaluación) (Vicente et al., 2014) contribuyen sin duda a alcanzar el objetivo de partida. Conseguirlo conlleva, por tanto, toda una serie de aspectos en los que el trabajo en grupos resulta fundamental: discusiones que aborden el interés y relevancia de los problemas o propuestas a abordar, elaboración de propuestas de resolución al respecto (formulación de preguntas, emisión de hipótesis y predicciones, diseño experimental, etc.) y finalmente, análisis y comunicación de resultados, cotejando los obtenidos por los distintos grupos de estudiantes y por la comunidad científica (Vilches y Gil-Pérez, 2011). 


\section{Necesidades de la enseñanza-aprendizaje por investigación cooperativa}

Conseguir el éxito de la "indagación cooperativa " recae en la consecución por parte de los actores (maestros y alumnos) de varios elementos de enseñanza-aprendizaje implicados en el proceso (Lederman et al., 2013).

En el caso de los docentes, foco de interés de este trabajo, es fundamental reconocer los conceptos fundamentales y las ayudas didácticas necesarias de forma estratégica, para aprovechar así los momentos de enseñanza que surjan durante la instrucción, entendidos estos como oportunidades en las que el aprendizaje del alumnado sobre un tema o idea resulta posible o más fácil (Woods-McConney et al., 2016). En este sentido, y refiriéndonos a las etapas del proceso indagatorio, es importante diferenciar entre los momentos de enseñanza planificados (en los cuales el alumnado refuerza y conecta el nuevo conocimiento adquirido de forma empírica) y aquellos que surgen de forma espontánea (en los que el docente debe elegir entre seguir el ritmo del plan de estudios o adaptarse a las necesidades del alumnado) (Haug, 2014).

Esta necesidad de modelar la práctica de investigación cooperativa (McNeill y Krajcik, 2008) requiere, por un lado, un conocimiento profundo del contenido, y por otro, de las habilidades y pedagogía necesarias para implementar con éxito este enfoque. Especialmente relevante es el uso de un lenguaje de investigación apropiado, que fomente el discurso cooperativo para promover la resolución de problemas y las habilidades de razonamiento en el alumnado (Gillies et al., 2013), a través de preguntas dirigidas por el docente que cumplan por igual la doble función comunicativa y social, ayudando a promover el pensamiento científico y establecer diferentes niveles de relaciones sociales (Oliveira, 2010).

Además de estas características, que pueden convertirse en factores limitantes, existen una serie de obstáculos que contribuyen a una pobre puesta en práctica de las experiencias de investigación. Particularmente clave es el grado de confianza del docente para que el alumnado pueda construir su aprendizaje por sí mismo, por lo que la orientación individualizada que ayude a superar los errores personales y enriquezca los planteamientos individuales iniciales ("fecundación cruzada de ideas" en palabras de Vilches y Gil-Pérez, 2011), cobra especial relevancia.

Otra de las dificultades a menudo asociadas es la preparación de la propia investigación, que requiere de un diseño cuidadoso que asegure un hilo conductor que aporte sentido al trabajo, orientando y haciendo una previsión fundamentada de la misma, para que no resulte en actividades independientes o completamente improvisadas (Vilches y GilPérez, 2011).

Todos estos aspectos ponen de manifiesto la necesidad de trabajar este tipo de enfoques desde la enseñanza básica (Fernández-Lozano et al., 2012), tal y como recomiendan instituciones de carácter internacional como la UNESCO (United Nations Educational Scientific and Cultural Organization [UNESCO], 2005), resultando fundamental para ello conocer las manifestaciones de los docentes al respeto para poder ofrecer una formación continuada que facilite la elaboración de propuestas que consideren esta modalidad de trabajo.

\section{Percepciones de docentes de Educación primaria en ejercicio sobre el uso de la investi- gación cooperativa}

A menudo existe una percepción positiva sobre las actitudes y visiones de los docentes en torno al uso de la investigación cooperativa. Trabajos como el de Gillies y Nichols, (2015) reflejan cómo los maestros y maestras perciben este enfoque como un motor para el proceso de aprendizaje del alumnado, que les acerca temáticas del mundo real que 
contribuyen a un aprendizaje más significativo. No obstante, sí se identifica un grado de preocupación referente a la estructuración del proceso de investigación, asociado a las herramientas necesarias para desafiar el pensamiento del alumnado y ayudarle en su aprendizaje para alentar la discusión. Este factor es altamente relevante, ya que el grado de confianza determina las probabilidades de probar nuevas estrategias e ideas al enfrentarse a situaciones desafiantes en el aula, frecuentes en los procesos de investigación (Marshall et al., 2009).

La oportunidad de trabajar cooperativamente también es vista como positiva, ya que consideran que aporta confianza a su alumnado y contribuye a las discusiones y al trabajo en equipo para construir conjuntamente nuevas comprensiones (Gillies y Nichols, 2015).

Entre las principales preocupaciones, además de aquellas relacionadas con la gestión del aula o las restricciones propias del sistema educativo (Romero-Ariza et al., 2020; Silm et al., 2015), destacamos las propias asociadas a los desafíos que impone la investigación, como el hecho de reconducir las discusiones del alumnado o las limitaciones impuestas por los requisitos de evaluación (Gillies y Nichols, 2015).

Aunque existen numerosas referencias en la literatura que reflejan cómo estas percepciones generales sobre el uso del enfoque de investigación cooperativa pueden estar asociados a factores demográficos, culturales o propios del perfil profesional docente, entre otros, sí queremos resaltar el trabajo de Marshall et al. (2009) por ser de los pocos estudios que incluye, además de los ya mencionados, el género como posible factor determinante en la enseñanza de las ciencias por investigación.

\section{"Andalucía Profundiza" como contexto de la investigación}

El programa de profundización de conocimientos "Andalucía Profundiza" consiste en la realización de proyectos de investigación cooperativos dirigidos a favorecer la estimulación del aprendizaje y la iniciativa del alumnado. Esta aproximación pretende involucrar al alumnado en el trabajo en problemas reales en pequeños grupos e investigar sobre las posibles soluciones, con la participación del docente como facilitador del proceso. Se trata de un programa que representa una oportunidad para innovar metodologías y estrategias de aprendizaje, así como motivar al alumnado que muestra un especial interés hacia el conocimiento y la investigación.

El programa, que comenzó su andadura en el curso académico 2009/2010 con 120 proyectos y 1700 alumnos, ha incrementado su participación durante estos diez años hasta en un total de cerca de 2950 proyectos (según datos de la página oficial), con 457 proyectos registrados en el curso 2018/2019 y 6855 alumnos.

Implementado en centros de carácter público dependientes de la Consejería de Educación y Deporte de la Junta de Andalucía, promueve un abanico de proyectos orientados a estudiantes de Educación Primaria y de Educación Secundaria Obligatoria, realizados en horario no lectivo, cuyos objetivos, según contempla la normativa reguladora correspondiente (Consejería de Educación y Deporte Junta de Andalucía [CEDJA], 2020) son los siguientes:

- Promover la profundización del conocimiento en el alumnado con interés en avanzar más allá de lo que se plantea de manera ordinaria en el aula.

- Estimular en el alumnado el interés por la investigación y la adquisición de estrategias que le permita la mejora de sus competencias a lo largo de la vida.

1 Puede accederse a la web del programa desde la siguiente dirección: http://profundiza.org 
- Fomentar la iniciativa personal y la autonomía del alumnado, fomentando la capacidad de emprendimiento y estimulando la creatividad, innovación y originalidad.

- Fomentar nuevas prácticas docentes, favoreciendo metodologías innovadoras para el desarrollo de las competencias clave del alumnado.

- Dar a conocer buenas e innovadoras prácticas docentes en el terreno de la investigación aplicada a la educación.

La participación del alumnado, al igual que la del profesorado, tiene un carácter voluntario, basado en el interés y la disposición positiva hacia el desarrollo de proyectos de investigación.

Los criterios de selección de los docentes participantes contemplan la originalidad y calidad de las propuestas de investigación presentadas. Se consideran, además de la experiencia profesional, la experiencia acreditada en la aplicación en el aula de proyectos de investigación como enfoque de enseñanza-aprendizaje (Consejería de Educación y Deporte Junta de Andalucía [CEDJA], 2020).

\section{Metodología}

\section{Participantes}

En esta investigación han participado un total de 75 maestros y maestras en ejercicio procedentes de diferentes centros educativos ubicados en la zona de Andalucía Oriental. Es en todo caso una muestra de conveniencia o accidental (Cohen et al., 2007), ya que se trata de participantes que, voluntariamente, aceptaron participar en la investigación. Por otro lado, no puede considerarse representativa de los maestros y maestras de Educación Primaria en general, ya que superaron un proceso inicial de selección en base a su experiencia previa en la práctica de proyectos de investigación (requisito del programa Profundiza).

El perfil de los participantes estaba representado por un $61 \%$ de mujeres, con una amplia experiencia docente (superior a 10 años para más del 70\% de la muestra). Además, en la caracterización se les preguntó para la enseñanza de qué materias estaba enfocada su formación inicial. Una ampliación de estos datos se recoge en la tabla 1, en la que, además, aparecen desglosados por género la experiencia docente y la formación inicial.

La distribución por género y su relación con la experiencia docente se encuentra en consonancia con los resultados recogidos en el "Informe sobre el Panorama de la Educación con los indicadores de la OCDE" (Instituto Nacional de Evaluación Educativa, 2018), que hace referencia a los resultados obtenidos en el "Estudio Internacional sobre Enseñanza y Aprendizaje" (TALIS) (Organisation for Economic Co-operation and Development [OECD], 2019).

\section{Instrumento de toma de datos}

El cuestionario planteado, cuyas respuestas siguen una escala tipo Likert (1, "totalmente en desacuerdo" y 4, "totalmente de acuerdo"), es una adaptación del proyecto europeo PRIMAS (Engeln et al., 2013). ${ }^{2}$ Los 23 ítems analizados se han estructurado en diferentes bloques, todos ellos relacionados con la implementación del enfoque de investigación y estrategias de trabajo cooperativo como parte de las metodologías activas.

2 The PRIMAS Project: promoting inquiry-based learning (IBL) in mathematics and science education across Europe (www.primasproject.eu) 
Tabla 1. Caracterización del perfil de los participantes

\begin{tabular}{|c|c|c|c|}
\hline \multicolumn{2}{|r|}{ Aspecto } & $\mathbf{N}$ & $\%$ \\
\hline Género & $\begin{array}{l}\text { Hombre } \\
\text { Mujer }\end{array}$ & $\begin{array}{l}29 \\
46\end{array}$ & $\begin{array}{l}38.7 \\
61.3\end{array}$ \\
\hline \multirow{8}{*}{ Experiencia docente } & Menos de 5 años & 5 & 6.7 \\
\hline & Hombre & $\begin{array}{l}2 \\
3\end{array}$ & $\begin{array}{l}2.7 \\
4.0\end{array}$ \\
\hline & Entre 5 y 10 años & 15 & 20.0 \\
\hline & Hombre & $\begin{array}{l}6 \\
9\end{array}$ & $\begin{array}{r}8.0 \\
12.0\end{array}$ \\
\hline & Entre 10 y 20 años & 39 & 52.0 \\
\hline & Hombre & $\begin{array}{l}13 \\
26\end{array}$ & $\begin{array}{l}17.3 \\
34.7\end{array}$ \\
\hline & Más de 20 años & 16 & 21.3 \\
\hline & Hombre & $\begin{array}{l}8 \\
8\end{array}$ & $\begin{array}{l}10.7 \\
10.7\end{array}$ \\
\hline \multirow{4}{*}{ Formación inicial } & Educación Primaria & 60 & 80.0 \\
\hline & Hombre & $\begin{array}{l}19 \\
41\end{array}$ & $\begin{array}{l}25.3 \\
54.7\end{array}$ \\
\hline & Otras (*) & 15 & 20.0 \\
\hline & Hombre & $\begin{array}{r}11 \\
4\end{array}$ & $\begin{array}{r}14.7 \\
5.3\end{array}$ \\
\hline
\end{tabular}

(*) En esta categoría los participantes especificaron Ciencias Naturales, Informática, Matemáticas y Tecnología. Considerando que más del $20 \%$ de la muestra presenta una experiencia docente superior a 20 años, es posible que pudiesen, en su momento, incorporarse a la docencia en Educación Primaria con una formación inicial (licenciaturas, grados e ingenierías), diferente a los estudios de magisterio.

\section{Recogida, tratamiento y análisis de datos}

Los datos se recogieron (en formato papel) tras la realización de las IX Jornadas Andalucía Profundiza "Cooperar, investigar, aprender" (área de Andalucía Oriental), celebradas en Málaga en el año 2019. Para el análisis de los resultados se calculó el valor promedio otorgado por los participantes (entre 0 y 4 ) y las desviaciones estándar para cada ítem. Para determinar la existencia o no de diferencias significativas entre los perfiles de la muestra, tras verificar que los datos obtenidos no seguían una distribución normal, se realizó un estudio estadístico no paramétrico con el programa RStudio, mediante el análisis de la $\mathrm{H}$ de Kruskal-Wallis para muestras no pareadas e independientes y la prueba $\mathrm{U}$ de MannWhitney para muestras pareadas e independientes, estipulando un valor del coeficiente de correlación $p$ inferior 0.05 para admitir la existencia de diferencias significativas.

\section{Resultados}

Los resultados obtenidos se han organizado en torno a las dos ideas principales analizadas en este trabajo. Por un lado, aquellos ítems que corresponden a aspectos generales del uso de metodologías activas (ítems 1-14); por otro, aquellos más específicos que hacen referencia a características propias del enfoque de investigación cooperativa. (ítems 15-23). Para cada uno de los ítems, presentamos los resultados estadísticos, resaltando aquellos que hemos considerado relevantes por cumplir con el exigente criterio de que el valor de la media fuese igual o superior a 3.5 para ítems redactados en positivo, y menor a 1.5 para ítems redactados en negativo. 


\section{Uso de metodologías activas de enseñanza-aprendizaje}

Los resultados en cuanto a la conexión con la motivación del alumnado y la dependencia del conocimiento en la práctica de metodologías activas de enseñanza-aprendizaje, se recogen en la tabla 2 (ítems 1-4).

Tabla 2. Resultados relacionados con ventajas educativas y requisitos para el uso de metodologías activas

\begin{tabular}{|c|c|c|c|c|}
\hline & Media & $\mathrm{DE}$ & Carácter \\
\hline \multicolumn{5}{|c|}{ Las metodologías activas de enseñanza-aprendizaje... } \\
\hline 1 & $\begin{array}{l}\text {...son adecuadas para afrontar problemas de motivación del } \\
\text { alumnado. }\end{array}$ & 3.72 & 0.45 & + \\
\hline 2 & $\begin{array}{l}\text {...son apropiadas para abordar problemas de aprendizaje del } \\
\text { alumnado }\end{array}$ & 3.57 & 0.57 & + \\
\hline 3 & $\begin{array}{l}\text {...requieren que el alumnado tenga un amplio conocimiento } \\
\text { inicial. }\end{array}$ & 2.01 & 0.76 & + \\
\hline 4 & ...no son efectivas en alumnado de bajo rendimiento. & 1.78 & 0.72 & - \\
\hline
\end{tabular}

Así, la figura 1 muestra cómo la mayoría de los docentes encuestados considera que existe una relación directa positiva (aquellas que entren en la categoría "de acuerdo" y "totalmente de acuerdo"), entre las metodologías activas y la motivación del alumnado (ítem 1), encontrándolas adecuadas para abordar problemas de aprendizaje (ítem 2).

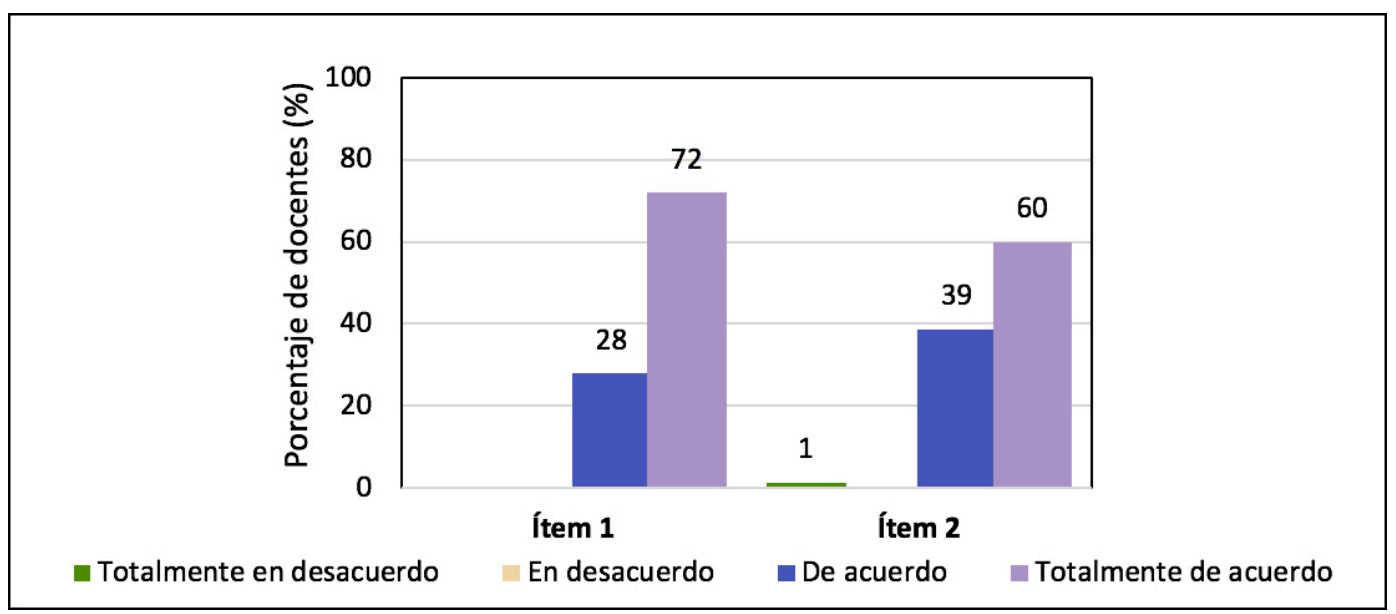

Figura 1. Resultados relacionados con la motivación y el aprendizaje del alumnado

Entre las dificultades referidas a la implementación de estas estrategias, los resultados obtenidos (tabla 3), muestran que los docentes participantes no consideran importantes las planteadas, ya que los valores medios obtenidos son mayores de 1.5 (para los ítems formulados de forma negativa) y menores de 3.5 (para los formulados de forma positiva). No obstante, se puede destacar que los tipos de evaluación habituales (ítem 13) y la ratio (ítem 14) aparecen como aspectos que plantean cierta dificultad para desarrollar metodologías activas. También hay que destacar los resultados de los ítems 7 y 9 , ya que teniendo en cuenta que se trata de docentes con experiencia en la implementación de proyectos de investigación (requisito del programa Andalucía Profundiza), se observa una cierta preocupación por la necesidad de acceder a programas específicos de formación y manifiesten no sentirse completamente seguros al utilizar metodologías activas. 
Tabla 3. Resultados relacionados con las dificultades en la aplicación de metodologías activas

\begin{tabular}{|c|c|c|c|c|}
\hline & & Media & DE & Carácter \\
\hline & uentro dificultades en la práctica de metodologías activas p & & & \\
\hline 5 & ...no dispongo de materiales didácticos adecuados. & 2.73 & 0.81 & - \\
\hline 6 & ...no están incluidas en los libros de texto. & 2.37 & 0.88 & - \\
\hline 7 & ...necesito acceso a programas de formación específicos. & 2.77 & 0.85 & + \\
\hline 8 & $\begin{array}{l}\text {...me preocupa que empeore el comportamiento de mi } \\
\text { alumnado. }\end{array}$ & 2.09 & 0.84 & + \\
\hline 9 & ...no me siento seguro con estas metodologías. & 2.12 & 0.81 & - \\
\hline 10 & $\begin{array}{l}\text {...me preocupa que mi alumnado se pueda sentir perdido y } \\
\text { frustrado en su aprendizaje. }\end{array}$ & 2.12 & 0.79 & + \\
\hline 11 & ...en el currículo no se fomenta. & 2.53 & 0.81 & - \\
\hline 12 & ...no hay tiempo suficiente para cumplir la programación. & 2.68 & 0.87 & - \\
\hline 13 & $\begin{array}{l}\text {...las evaluaciones a las que se enfrenta mi alumnado no } \\
\text { tienen en cuenta todos los aspectos desarrollados con estas } \\
\text { metodologías. }\end{array}$ & 2.81 & 0.87 & - \\
\hline 14 & $\begin{array}{l}\text {...habitualmente el número de alumnos por clase es muy } \\
\text { elevado para que estas metodologías sean efectivas. }\end{array}$ & 2.87 & 1.03 & + \\
\hline
\end{tabular}

\section{Estrategias de investigación cooperativa}

Los resultados que recogen aspectos relacionados con el uso del enfoque de investigación cooperativa se presentan en la tabla 4, reflejando cómo en general, existe un alto grado de aceptación sobre la importancia de este enfoque y las habilidades que permite desarrollar.

Tabla 4. Resultados relacionados sobre el uso del enfoque de investigación cooperativa

\begin{tabular}{|c|c|c|c|c|}
\hline & Media & $\mathrm{DE}$ & Carácter \\
\hline \multicolumn{5}{|c|}{ En cuanto a las metodologías activas de e-a, considero que... } \\
\hline 15 & $\begin{array}{l}\text {...hay que proporcionar oportunidades al alumnado para que } \\
\text { exprese y explique sus propias ideas. }\end{array}$ & 3.74 & 0.59 & + \\
\hline 16 & $\begin{array}{l}\text {...es necesario que los alumnos tengan discusiones/debatan } \\
\text { sobre el tema que estemos trabajando. }\end{array}$ & 3.64 & 0.63 & + \\
\hline 17 & $\begin{array}{l}\text {...es importante que el alumnado participe en el debate y } \\
\text { discusiones que se establezcan en clase. }\left({ }^{*}\right)\end{array}$ & 3.71 & 0.62 & + \\
\hline 18 & ...es importante realizar actividades prácticas. & 3.81 & 0.56 & + \\
\hline 19 & $\begin{array}{l}\text {...es importante que los alumnos realicen experimentos/ } \\
\text { investigaciones siguiendo mis instrucciones. }\end{array}$ & 3.40 & 0.70 & + \\
\hline 20 & $\begin{array}{l}\text {...es fundamental que el alumnado obtenga conclusiones a } \\
\text { partir de los experimentos/simulaciones/modelos que ellos } \\
\text { mismos han realizado. }\left({ }^{*}\right)\end{array}$ & 3.68 & 0.62 & + \\
\hline 21 & $\begin{array}{l}\text {...es fundamental que el alumnado diseñe sus propios } \\
\text { experimentos/investigaciones. }\end{array}$ & 3.52 & 0.67 & + \\
\hline 22 & $\begin{array}{l}\text {...el alumnado debe realizar investigaciones/experimentos para } \\
\text { comprobar sus propias ideas. }\end{array}$ & 3.63 & 0.63 & + \\
\hline 23 & ...es dificil gestionar el trabajo en grupo. & 2.43 & 0.83 & + \\
\hline
\end{tabular}

(*) Para estos ítems, el número de respuestas obtenidas fue inferior a 75 (72 para el ítem 7 y 74 para el ítem 10), calculando los estadísticos de acuerdo con estas poblaciones. 
Tal y como muestra la figura 2 , hasta un $97 \%$ de los participantes coinciden en la importancia de generar oportunidades de aprendizaje al alumnado, que le permitan exponer sus ideas propias (ítem 15), favoreciendo además las discusiones y/o debates (ítems 16 y 17), actividades fundamentales para analizar los datos empíricos y conectar los resultados con la teoría y los nuevos conocimientos adquiridos. Además, casi el mismo porcentaje de maestros y maestras (98\%) coincide en la importancia de realizar actividades de carácter práctico (ítem 18), siendo consciente de la importancia de obtener conclusiones (ítem 20) fomentando la autonomía del alumnado para diseñar sus propias experiencias y comprobar sus ideas propias (ítems $\mathbf{2 1}$ y 22).

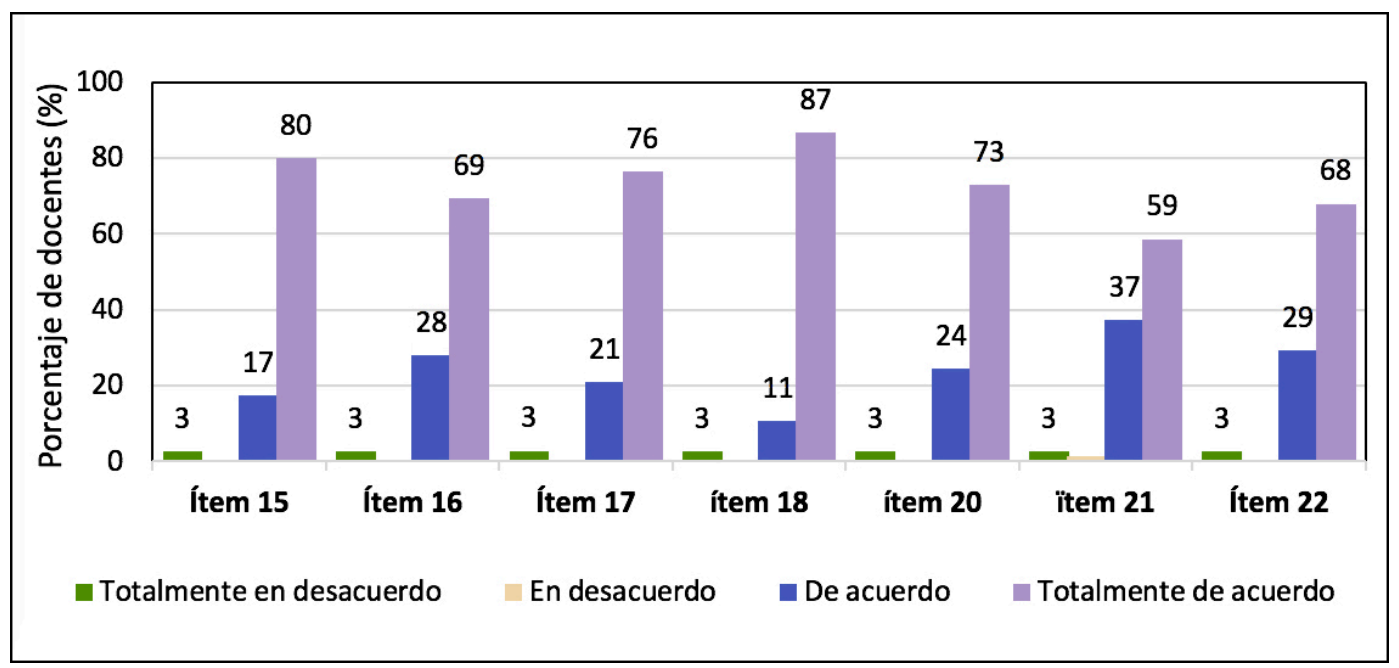

Figura 2. Resultados relacionados con el uso del enfoque de investigación cooperativa

Se aprecia una percepción de los docentes muy favorable sobre aquellos aspectos distintivos de la investigación, como son el diseño (ítem 21), la realización de experimentos (ítem 22) y la elaboración de conclusiones (ítem 20), y que estos aspectos tienen que ser realizados por los propios estudiantes. Al respecto también hay que resaltar que las actividades de investigación/indagaciones cerradas (ítem 19) no son altamente valoradas.

También se encuentra una percepción favorable a los aspectos más centrados en el aprendizaje cooperativo (ítems 16 y 17), no considerando los docentes que la gestión en el aula del trabajo en grupo constituya un obstáculo importante (ítem 23).

\section{Relaciones de dependencia con el perfil profesional docente}

Para establecer las relaciones de dependencia entre percepciones manifestadas y el perfil profesional docente con el que se caracterizó la muestra (género, años de experiencia y formación inicial), realizamos un estudio estadístico para determinar la existencia o no de diferencias significativas. Los resultados al respecto pueden ayudar a identificar grupos docentes con necesidades específicas de apoyo para abordar enfoques de enseñanza-aprendizaje centrados en el alumnado. De este modo, solo se ha encontrado una relación de dependencia del género para los ítems 9 y 23, cuyos valores se recogen en la tabla 5. 
Tabla 5. Uso de metodologías activas y dependencia de género

\begin{tabular}{|c|c|c|c|c|}
\hline \multicolumn{2}{|r|}{ Las metodologías activas de enseñanza-aprendizaje... } & p & Media & $\mathrm{DE}$ \\
\hline \multirow[b]{2}{*}{9} & ...no me siento seguro con estas metodologías. & 0.01 & 2.12 & 0.81 \\
\hline & Hombre & & $\begin{array}{l}1.83 \\
2.30\end{array}$ & $\begin{array}{l}0.80 \\
0.76\end{array}$ \\
\hline \multirow[b]{2}{*}{23} & ...es difícil gestionar el trabajo en grupo. & 0.01 & 2.43 & 0.83 \\
\hline & Hombre & & $\begin{array}{l}2.14 \\
2.61\end{array}$ & $\begin{array}{l}0.88 \\
0.74\end{array}$ \\
\hline
\end{tabular}

*Percepciones estadísticas diferentes $(p<0.05)$

Esta dependencia puede observarse gráficamente en el diagrama de cajas (figura 3), en el que se representan los valores máximos y mínimos alcanzados para cada muestra, junto con los percentiles $25 \%$ y $75 \%$, con el punto " $x$ " indicando el valor medio. Estos gráficos ponen de manifiesto cómo las maestras se sienten ligeramente más inseguras a la hora de aplicar metodologías activas (ítem 9), considerando además una mayor dificultad en la gestión del trabajo en grupo (ítem 23).
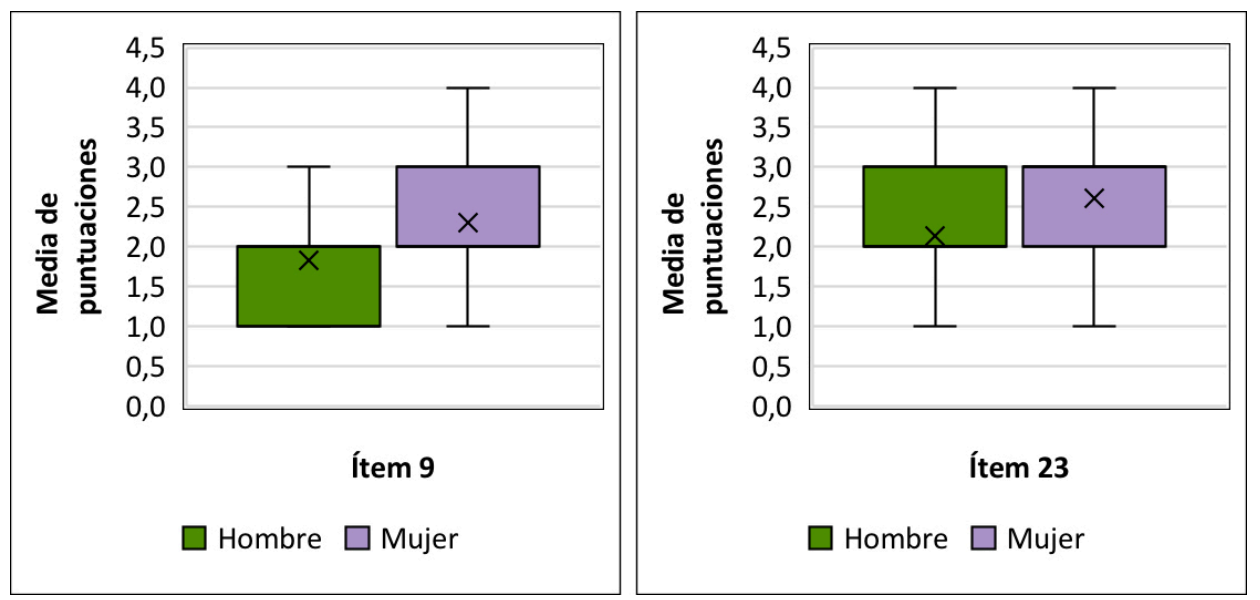

Figura 3. Dependencia con la variable género

\section{Discusión}

En este trabajo, abordamos desde una perspectiva cuantitativa las percepciones de maestros y maestras participantes en el programa Andalucía Profundiza sobre las metodologías activas de enseñanza-aprendizaje y el uso del enfoque de investigación cooperativa, así como la posible existencia de relaciones de dependencia con los perfiles profesionales docentes.

En cuanto a la percepción general y la relación entre las creencias sobre las habilidades que promueve este tipo de metodologías, observamos un claro consenso en torno a sus efectos positivos, tal y como establecen Gillies y Nichols (2015), considerando los participantes su repercusión en la motivación hacia el aprendizaje de la ciencia, si bien los resultados son moderados respecto a las necesidades para garantizar el éxito de su aplicación (amplio conocimiento inicial y alto rendimiento académico de los estudiantes).

Más satisfactorios son los resultados obtenidos sobre la importancia de fomentar la investigación en el aula, con un alto grado de aceptación de aspectos distintivos de la investigación (el diseño, la realización de experimentos o la obtención de conclusiones) y del trabajo 
cooperativo. En este sentido, el hecho de tratarse de una muestra de participantes acostumbrada a poner en prácticas proyectos de investigación cooperativa hace que su visión y percepción al respecto está notablemente influenciada. Es por ello quizás que los resultados obtenidos sobre las dificultades encontradas a la hora de manejar este tipo de enfoques didácticos no resaltan especialmente, si bien todavía existe una cierta inseguridad en su aplicación y en la gestión del trabajo en grupo.

Finalmente, observamos una dependencia de género en cuanto al uso de metodologías activas y de estrategias cooperativas, manifestándose las maestras más inseguras que los maestros en su aplicación y considerando más difícil la gestión del trabajo en grupo. Estos resultados podrían explicarse por la desigual distribución de género y experiencia docente (con una mayor representación de mujeres respecto a hombres con entre 10 y 20 años de experiencia), teniendo en cuenta el carácter innovador de estas metodologías y su inclusión relativamente reciente en los programas de formación docente. Al margen de estos dos aspectos, los demás resultados son acordes con los obtenidos por Marshall et al. (2009), que describen una independencia entre la variable género y las creencias de docentes sobre el enfoque de investigación.

\section{Conclusiones, limitaciones y perspectivas de futuro}

Aunque existen varios instrumentos para analizar las percepciones de los maestros y maestras de primaria acerca del uso de metodologías activas de enseñanza-aprendizaje, la adaptación del cuestionario utilizado permite además relacionarlas con las estrategias de trabajo cooperativo, a menudo interconectadas, abordando así las dificultades y actitudes ante la aplicación de un enfoque de investigación cooperativo en el aula de ciencias y considerando los posibles escenarios al respecto.

No obstante, encontramos ciertas limitaciones, referidas especialmente a la necesidad de realizar un estudio longitudinal que profundice en el cambio en cuanto a las percepciones antes, durante, y tras la implementación de los proyectos Andalucía Profundiza, en línea con las recomendaciones de Bryan (2012).

Los resultados obtenidos son satisfactorios y muestran una percepción positiva de los maestros/as en activo sobre el uso de estrategias y dinámicas relacionadas con el aprendizaje cooperativo o la indagación científica y el aprendizaje basado en proyectos, convirtiéndose en una ventaja a la hora de ser incorporadas en los proyectos Andalucía Profundiza. Sin embargo, todavía se requieren de procesos de formación y concienciación para ser implementadas de forma generalizada por el profesorado en ejercicio (LupiónCobos y Martín-Gámez, 2016). Por tanto, consideramos necesarios el desarrollo de cursos y seminarios de formación específicos sobre enseñanza por investigación cooperativa, que aporten modelos de ejemplificación de buenas prácticas y proporcionen a los maestros y maestras un entorno de apoyo en el aprendizaje docente, y que puedan verse reflejados en una mejora de la calidad de la docencia impartida.

\section{Agradecimientos}

Este trabajo forma parte del proyecto de I+D de Excelencia "Desarrollo de competencias en problemas de la vida diaria mediante prácticas científicas de argumentación, indagación y modelización en enseñanza secundaria y universitaria (EDU2017-82197-P), financiado por el Ministerio de Ciencia, Innovación y Universidades en 2017. La Dra. García-Ruiz agradece al Plan Propio de la Universidad de Málaga la "Ayuda para incorporación de doctores" recibida. 


\section{Referencias bibliográficas}

Bryan, L. A. (2012). Research on science teachers belifs. En B. J. Fraser, K. G. Tobin, y C. J. McRobbie (Eds.), Second International Handbook of Science Education (pp. 477-495). Springer. DOI: https://doi.org/10.1007/978-1-4020-9041-7

Cohen, L., Manioin, L. y Morrison, K. (2007). Research methods in education. Sixth Edition. Routledge. DOI: https://doi.org/10.1080/19415257.2011.643130

Comeaux, P. y Huber, R. (2001). Students as scientists: using interactive technologies and collaborative inquiry in an environmental science project for teachers and their students. Journal of Science Teacher Education, 12, 235-252. DOI: https://doi. org/10.1023/ A:1014226110211

Consejería de Educación y Deporte Junta de Andalucía [CEDJA]. (2020). Instrucciones de 20 de Enero de 2020, de la Dirección General de Atención a la Diversidad, Participación y Convivencia Escolar, por la que se regula el funcionamiento del programa de profundización de conocimientos "Andalucía Profundiza", en los centros.

Couso, D. (2014). De la moda de "aprender indagando" a la indagación para modelizar: una reflexión crítica. En M. A. Herás, A. Lorca, B. Vázquez, A. Wamba, y R. Jiménez (Eds.), Investigación y transferencia para una educación en ciencias: Un reto emocionante (pp. 1-28). Servicio de Publicaciones Universidad de Huelva.

Domènech-Casal, J. (2018). Aprendizaje Basado en Proyectos en el marco STEM. Componentes didácticas para la competencia científica. Ápice. Revista de Educación Científica, 2(2), 29-42. DOI: https://doi.org/10.17979/arec.2018.2.2.4524

Durando, M., Sjøberg, S., Gras-Velázquez, A., Leontarai, I., Martín-Santolaya, E. y Tasiopoulou, E. (2019). Teacher training and IBSE practice in Europe - A European Schoolnet overview. In European Schoolnet (p. 33). European Schoolnet.

Engeln, K., Euler, M. y Maass, K. (2013). Inquiry-based learning in mathematics and science: A comparative baseline study of teachers' beliefs and practices across 12 European countries. ZDM - International Journal on Mathematics Education, 45(6), 823-836. DOI: https://doi.org/10.1007/s11858-013-0507-5

Fernández-Lozano, M. P., González-Ballesteros, M. y De-Juanas, A. (2012). El alcance del trabajo cooperativo en el aula desde el punto de vista de los maestros de Educación Primaria. Electronic Journal of Research in Educational Psychology, 10, 171-194.

Fiore, S. M., Rosen, M. A., Smith-Jentsch, K. A., Salas, E., Letsky, M. y Warner, N. (2010). Toward an understanding of macrocognition in teams: Predicting processes in complex collaborative contexts. Human Factors: The Journal of the Human Factors and Ergonomics Society, 52(2), 203-224. DOI: https://doi. org/10.1177/0018720810369807

Gillies, R. M. y Nichols, K. (2015). How to support primary teachers' implementation of inquiry: teachers' reflections on teaching cooperative inquiry-based science. Research in Science Education, 45(2), 171-191. DOI: https://doi.org/10.1007/ s11165-014-9418-x

Gillies, R. M., Nichols, K., Burgh, G. y Haynes, M. (2013). Primary students' scientific reasoning and discourse during cooperative inquiry-based science activities. International Journal of Educational Research, 63, 127-140. DOI: https://doi. org/10.1016/j.ijer.2013.01.001 
Haug, B. S. (2014). Inquiry-based Science: turning teachable moments into learnable moments. Journal of Science Teacher Education, 25(1), 79-96. DOI: https://doi. org/10.1007/s10972-013-9375-7

Hesse, F., Care, E., Buder, J., Sassenberg, K. y Griffin, P. (2015). A framework for teachable collaborative problem solving skills. En P. Griffin y E. Care (Eds.), Assessment and teaching of 21st century skills (pp. 37-56). Springer. DOI: https://doi. org/10.1007/978-94-017-9395-7_2

Instituto Nacional de Evaluación Educativa. (2018). Panorama de la educación. Indicadores de la OCDE 2018. Informe español. Ministerio de Educación y Formación Profesional.

Lederman, N. G., Lederman, J. S. y Antink, A. (2013). Nature of science and scientific inquiry as contexts for the learning of science and achievement of scientific literacy. International Journal of Education in Mathematics, Science and Technology, 1, 138-147. DOI: https://doi.org/10.18404/ijemst.19784

Lupión-Cobos, T. y Martín-Gámez, C. (2016). Desarrollo profesional docente de profesorado de secundaria en una experiencia de innovación mediante investigaciones escolares. Revista Eureka Sobre Enseñanza y Divulgación de Las Ciencias., 13, 686-704. DOI: https://doi.org/10.25267/rev_eureka_ensen_divulg_cienc.2016.v13.i3.13

Marshall, J. C., Horton, R., Igo, B. L. y Switzer, D. M. (2009). K-12 science and mathematics teachers' beliefs about and use of inquiry in the classroom. International Journal of Science and Mathematics Education, 7(3), 575-596. DOI: https://doi.org/10.1007/ s10763-007-9122-7

Marshall, J. C., Smart, J. y Horton, R. M. (2009). The design and validation of equip: an instrument to assess inquiry-based instruction. International Journal of Science and Mathematics Education, 8, 299-321.

McNeill, K. L., y Krajcik J. S. (2008). Scientific explanations: characterizing and evaluating the effects of teachers' instructional practices on student learning. Journal of Research in Science Teaching, 45(1), 53-78. DOI: https://doi.org/10.1002/tea.20201

Mioduser, D. y Betzer, N. (2008). The contribution of project-based-learning to highachievers' acquisition of technological knowledge and skills. International Journal of Technology and Design Education, 18(1), 59-77. DOI: https://doi.org/10.1007/ s10798-006-9010-4

Oliveira, A. W. (2010). Improving teacher questioning in science inquiry discussions through professional development. Journal of Research in Science Teaching, 47(4), 422-453. DOI: https://doi.org/10.1002/tea.20345

Organisation for Economic Co-operation and Development [OECD]. (2017). PISA 2015 Assessment and analytical framework. Science, reading, mathematic, financial literacy and collaborative problem solving (revised edition). OECD Publishing. DOI: https://doi.org/https://doi.org/10.1787/9789264281820-en

Organisation for Economic Co-operation and Development [OECD]. (2019). TALIS 2018 results (Volume I): teachers and school leaders as lifelong learners. DOI: https://doi. org/10.1787/c9813405-ar

Osborne, J. (2011). Science teaching methods: a rationale for practices. School Science Review, 93(343), 93-103.

Osborne, J. y Dillon, J. (2008). Science education in Europe : critical reflections. In The Nuffield Foundation. DOI: https://doi.org/10.1080/10463280701728302 
Pérez, M. C. B. y Furman, M. (2016). What is a scientific experiment? The impact of a professional development course on teachers' ability to design an inquiry-based science curriculum. International Journal of Environmental and Science Education, 11, 1387-1401. DOI: https://doi.org/10.12973/ijese.2016.353a

Rocard, M., Csermely, P., Jorde, D., Walberg-Henriksson, H. y Hemmo, V. (2007). A renewed pedagogy for the future of Europe. In Economy and Society.

Romero-Ariza, M., Quesada, A., Abril, A. M., Sorensen, P. y Oliver, M. C. (2020). Highly recommended and poorly used: English and Spanish science teachers 'views of Inquiry-based Learning (IBL) and its enactment. Eurasia Journal of Mathematics, Science and Technology Education, 16(1), 1-16.

Silm, G., Tittsaar, K., Pedaste, M., Zacharia, Z. C. y Papaevripidou, M. (2015). Teacher's readiness to use inquiry-based learning: an investigation of teachers' sense of efficacy and attitudes toward inquiry-based learning. Science Education International, 28(4), 315-325.

Sjøberg, S. (2019). Critical Perspectives on Inquiry-Based Science Education (IBSE) in Europe. Position Paper Written for EUN Partnership, European Schoolnet, March.

Song, Y. (2018). Improving primary students' collaborative problem solving competency in project-based science learning with productive failure instructional design in a seamless learning environment. Educational Technology Research and Development, 66(4), 979-1008. DOI: https://doi.org/10.1007/s11423-018-9600-3

Ucan, S., y Webb, M. (2015). Social regulation of learning during collaborative inquiry learning in science: how does it emerge and what are its functions? International Journal of Science Education, 37(15), 2503-2532. DOI: https://doi.org/10.1080/09 500693.2015.1083634

United Nations Educational Scientific and Cultural Organization [UNESCO]. (2005). Towards knowledge societies. En UNESCO World Report. DOI: https://doi.org/10.1017/ CBO9781107415324.004

Vicente, F., López, M. y Vallés, C. (2014). Los rincones de trabajo como estrategia en la formación de maestros para la enseñanza de ciencias y su didáctica. Tendencias Pedagógicas, 23, 109-126.

Vigotsky, L. S. (1978). Mind in society. The development of higher psychological processes (M. Cole, V. John-Steiner, S. Scribner, y E. Souberman (eds.)). Harvard University Press. DOI: https://doi.org/10.3928/0048-5713-19850401-09

Vilches, A., y Gil-Pérez, D. (2011). El trabajo cooperativo en las clases de ciencias: una estrategia imprescindible pero aún infrautilizada. Alambique. Didáctica de Las Ciencias Experimentales, 69, 73-79.

Woods-McConney, A., Wosnitza, M. y Sturrock, K. L. (2016). Inquiry and groups: Student interactions in cooperative inquiry-based science. International Journal of Science Education, 38(5), 842-860. DOI: https://doi.org/10.1080/09500693.2016.1169454 
